\title{
Time to Rethink the Default Settings in Neuroscience: Hormonal Transition Periods as Natural Experiments and Why Sex Matters.
}

\author{
Rachel G. Zsido ${ }^{a}$,Julia Sacher ${ }^{b}$
}

\begin{abstract}
Diversity drives scientific discovery. Yet, many basic and clinical neuroscience studies fail to include equal numbers of females in their samples, and even fewer present sex-specific analysis of their data. We propose the following strategies to overcome this bias: (1) increase numbers of female study participants, (2) consider sex as a primary variable, and (3) when justified, study all-female samples to provide a more indepth understanding of female-specific experiences such as the menstrual cycle as well as sex-specific risk trajectories and pathologies. In our research program, we study the influence of sex and sex hormones on brain states in health and disease. We strive to explain the mechanisms underlying the unique vulnerability of women to depression and dementia. Our ultimate goal is to improve brain health for both sexes. By applying scientific expertise in neuropharmacology, quantitative neurochemical imaging and sex differences to traditional research questions in the cognitive sciences, we provide novel perspectives on the diversity of human cognition and brain plasticity.
\end{abstract}

Knowledge gaps and missing data are often the primary driving force behind the development of scientific breakthroughs. The curiosity and ur-

a Max Planck Institute for Human Cognitive and Brain Science

Emotions and NeuroimaGinG (EGG) Lab, Max Planck Institute for Human Cognitive and Brain Sciences, Leipzig, Germany

Department of Neurology, Max Planck Institute for Human Cognitive and Brain Sciences, Leipzig, Germany

Max Planck School of Cognition, Leipzig, Germany.

b Max Planck Institute for Human Cognitive and Brain Science

Emotions and NeuroimaGinG (EGG) Lab, Max Planck Institute for Human Cognitive and Brain Sciences, Leipzig, Germany

Department of Neurology, Max Planck Institute for Human Cognitive and Brain Sciences, Leipzig, Germany

Clinic for Cognitive Neurology, University of Leipzig, Leipzig, Germany. 
gency to discover answers when navigating an uncharted scientific landscape is viewed as a guiding principle for, and defining characteristic of, a scientist. But there remains a persistent lack of inclusion of the female population in research studies and standards, despite the translational relevance of these basic scientific data for over half the population. For example, the 1958 Baltimore Longitudinal Study of Aging on "normal human aging" did not include any women in the first 20 years. ${ }^{1}$ The 1982 Physicians' Health Study ${ }^{2}$ and 1973 Multiple Risk Factor Intervention Trial, ${ }^{3}$ two clinical trial studies on intervention of cardiovascular diseases, included thousands of men but no women. This systematic bias is not simply an issue of the past; even in 2016, only 54\% of public health studies, $43 \%$ of clinical medicine studies, and 30\% of basic biomedical research reported females and males. ${ }^{4}$

Of the studies that do include female subjects, sex is still not regularly considered as a primary factor and is often regressed out as a covariate (reviewed in Galea et al. ${ }^{5}$ ). Mersha and colleagues ${ }^{6}$ illustrated the potential oversights resulting from this approach by conducting both sex-specific and sex-combined analyses to test genomic associations with asthma risk. They found that over half of the key genetic variants were overlooked in sex-combined versus sex-specific analyses. Sex bias can exist unintentionally as well: Pirastu and colleagues ${ }^{7}$ investigated sex-specific study participation bias, revealing that large-scale, population-based studies are not always representative of the general population. Performing a genome-wide association study (GWAS) of sex with over 3 million participants, the authors demonstrated that a sex-differentiated participation bias in certain studies can result in spurious associations and inferences. All of these examples lead to lost opportunities in terms of informing basic scientific discovery; and without basic knowledge on the biological underpinnings of sex differences, we cannot address critical sex-driven differences in pathology. The current coronavirus disease 2019 (COVID-19) pandemic represents a topical example of the importance of recognizing and investigating such differences-there are prominent sex differences surfacing in mortality and

1 Shock, 1984.

2 Steering Committee of the Physicians' Health Study Research Group*, 1989.

3 Multiple Risk Factor Intervention Trial Research Group, 1982.

4 Sugimoto/Ahn/Smith/Macaluso/Larivière, 2019.

5 Galea/Choleris/Albert/McCarthy/Sohrabji, 2020.

6 Mersha/Martin/Myers/Kovacic/He/Lindsey/Sivaprasad/Chen/Hershey, 2015.

7 Pirastu/Cordioli/Nandakumar/Mignogna/Abdellaoui/Hollis/Kanai/Rajagopal/Parolo/ Baya, 2020. 
vulnerability that appear to characterize the disease ${ }^{8}$ but remain far from understood.

Another example of the critical potential to advance knowledge through proactive acknowledgement of sex differences is within technological breakthroughs, such as the use of artificial intelligence (AI) for healthcare and biomedical diagnosing. In a recent review, ${ }^{9}$ the authors emphasize how AI algorithms need to actively take into account sex and gender differences in order to make the most precise diagnoses and to recommend the most tailored and effective treatment, while avoiding unnecessary discrimination. Undesirable sex and gender discrimination often manifest unintendedly in training datasets. For example, Larrazabal and colleagues demonstrated the consequences of using sex-imbalanced medical imaging datasets, showing that algorithms performed significantly worse in female patients if the training dataset underrepresented female patients. ${ }^{10}$ And while the inverse was also true, the reality is that there is a critical lack of female participant availability in existing biomedical research datasets. Regardless of whether training and benchmarking datasets are biased by unrepresentative data or data that were never collected to begin with, the consequences can be devastating; whether that be for sex-specific pathology knowledge, clinical diagnosis purposes, or even chance of survival following an automobile accident. ${ }^{11}$

Neuroscience is particularly lagging ${ }^{12}$, with the male brain implicitly employed as the "default model" and only a minority of basic science and clinical neuroscience studies including a female sample. An analysis of neuroscience research articles in 2017 concluded that "male sex bias remains a persistent and perhaps even intensifying phenomenon in the neuroscience literature". ${ }^{13}$ Given robust sex differences in neuropsychiatric

8 Takehiro/Iwasaki, 2021; Global Health 5050, 2020; Guan/Ni/Hu/Liang/Ou/He/Liu/ Shan/Lei/Hui, 2020; Chen/Zhou/Dong/Qu/Gong/Han/Qiu/Wang/Liu/Wei, 2020; Wenham/Smith/Morgan, 2020; Chakravarty/Nair/Hammouda/Ratnani/Gharib/ Wagaskar/Mohamed/Lundon/Dovey/Kyprianou, 2020.

9 Cirillo/Catuara-Solarz/Morey/Guney/Subirats/Mellino/Gigante/Valencia/Rementeria/ Chadha, 2020.

10 Larrazabal/Nieto/Peterson/Milone/Ferrante, 2020.

11 See Holder, 2019, for female injury and fatality repercussions of historically using male-type crash test dummies; https:/www.bloomberg.com/news/articles/2019-07 -18/why-women-are-likelier-to-be-hurt-in-a-car-crash.

12 Beery/Zucker, 2011; Will/Proaño/Thomas/Kunz/Thompson/Ginnari/Jones/Lucas/Reavis/Dorris, 2017.

13 Will/Proaño/Thomas/Kunz/Thompson/Ginnari/Jones/Lucas/Reavis/Dorris, 2017. 
and neurodegenerative disease risk, ${ }^{14}$ brain anatomy ${ }^{15}$ as well as response to pharmaceuticals, ${ }^{16}$ neglect of women in neuroscientific research directly and detrimentally affects the health of women. This female underrepresentation may partially explain why women typically report more adverse event reactions to pharmacological interventions as compared to men. ${ }^{17}$ In the United States between 1997 and 2001, eight of the ten drugs that had to be withdrawn from the market had significantly higher health risks for women than for men. To identify biological contributors relevant to sexspecific effects, we must acknowledge and address this sex and gender data gap. ${ }^{18}$ Specifically, we need to promote scientific excellence by increasing the number of female participants, by considering sex and gender as primary variables in analyses, and-when justified-recruiting all-female samples to provide a more in-depth understanding of sex-specific risk trajectories and pathologies. This includes, but is not limited to, studies designed to investigate sex-specific mechanisms in topics where sex differences have already been observed, as well as female-specific experiences such as menstruation, hormonal contraceptive use, pregnancy/postpartum, and menopause - in which a male comparison group would not be scientifically meaningful. In this report, we will review some recent studies of the Emotion \& Neuroimaging (EGG) lab to investigate sex-specific risk trajectories on brain and cognitive health as well as the unique vulnerabilities of women to depression and dementia, including suggestions for future neuroscientific research on the intersection of sex and sex hormones in health and disease.

Sex differences in depression and dementia risk underscore the need to consider sex-specific mechanisms as a deliberate aim in human neuroimaging studies. In the EGG lab, Dr. Julia Sacher leads an interdisciplinary team of psychiatrists, psychologists and neuroscientists who focus on the distinct susceptibility of women to depressive symptoms across the lifespan. Through the use of multimodal neuroimaging techniques, our research aims to provide a mechanistic understanding of the interplay between sex hormones, brain structure and function, and female mental and

14 Kessler/Berglund/Demler/Jin/Koretz/Merikangas/Rush/Walters/Wang, 2003; Bromet/ Andrade/Hwang/Sampson/Alonso/De Girolamo/De Graaf/Demyttenaere/Hu/Iwata, 2011; Ruitenberg/Ott/van Swieten/Hofman/Breteler, 2001; Carter/Resnick/Mallampalli/Kalbarczyk, 2012.

15 Liu/Seidlitz/Blumenthal/Clasen/Raznahan, 2020.

16 LeGates/Kvarta/Thompson, 2019.

17 Simon, 2005; Tharpe, 2011; Light/Lovell/Butt/Fauvel/Holdcroft, 2006.

18 Perez, 'Invisible Women', 2019; D'Ignazio/Klein, 2020; https://data2x.org/. 
cognitive health. A major focus of the lab is to identify what neurochemical changes occur in the brain during hormonal transition phases across the lifespan, and determine if these changes may explain why women have the highest vulnerability towards depressed mood when sex hormones fluctuate and decline rapidly. ${ }^{19}$ Positron emission tomography (PET) can be used for in-vivo quantification of such neurochemical changes, ${ }^{20}$ potentially allowing for early identification of biomarkers of mood disorders. One critical hormonal transition period is the postpartum period, during which estrogen levels drop up to 1000 -fold after delivery. ${ }^{21}$ This immense hormonal disruption and the subsequent neurochemical consequences may underlie the "baby blues" experienced by up to $80 \%$ of mothers within days of delivery, or the more severe clinical form (postpartum depression [PPD]) experienced by approximately $15 \%$ of mothers and occurring within a month of delivery.

Sacher and colleagues used PET to measure monoamine oxidase A (MAO-A) levels in women who had given birth within 4-6 days, and found that greater MAO-A levels and activity were associated with both lower estrogen levels and greater symptom severity. ${ }^{22}$ Women who developed PPD within a month had sustained high levels of MAO-A. ${ }^{23}$ This interaction between MAO-A density, depressed mood and sex hormones is critical, as MAO-A metabolizes key monoamine neurotransmitters, such as serotonin, and excessive removal of such monoamines can have detrimental effects. This project provides insight into the uniquely female-specific neurobiology of the early postpartum period that remains underrepresented in neuroscientific literature. And while studies in these populations are challenging, the concerns can be addressed with thoughtful study design (see suggestions in Zsido et al. ${ }^{24}$ ) and the outcomes have clear implications for informing treatment options. For example, as treatment in these women should be compatible with breastfeeding, the results of this study suggest taking dietary supplements of monoamine precursors to maintain sufficient balance of monoamines during the early postpartum period.

19 Zsido/Villinger/Sacher, 2017; Deecher/Andree/Sloan/Schechter, 2008; Freeman/Sammel/Boorman/Zhang, 2014; Frokjaer/Pinborg/Holst/Overgaard/Henningsson/Heedel Larsen/Jensen/Agn/Nielsen, 2015.

20 Zsido/Villinger/Sacher, 2017.

21 O'Hara/Schlechte/Lewis/Wright, 1991; O'Hara/Swain, 1996; Nott/Franklin/Armitagel Gelder, 1976.

22 Sacher/Wilson/Houle/Rusjan/Hassan/Bloomfield/Stewart/Meyer, 2010.

23 Sacher/Rekkas/Wilson/Houle/Romano/Hamidi/Rusjan/Fan/Stewart/Meyer, 2015.

24 Zsido/Villinger/Sacher, 2017. 
Beyond these extensive hormonal shifts during the postpartum period, there are also more subtle hormonal fluctuations that can pre-dispose women to depressive symptoms, such as during the menstrual cycle. Approximately $8 \%$ of women during their reproductive years will develop premenstrual dysphoric disorder (PMDD), which is characterized by symptoms such as irritability, depression and anxiety. ${ }^{25}$ As these core symptoms occur exclusively in the late luteal phase before menstruation and when hormones are rapidly decreasing, the immediate assumption was that ovarian hormones such as estradiol were independently driving the depressed mood. Indeed, previous work has shown that fluctuations in estradiol levels around a woman's own mean is the strongest risk factor for developing symptoms. ${ }^{26}$ Yet, women suffering from PMDD do not have differences in absolute levels of ovarian hormones compared to healthy controls, ${ }^{27}$ suggesting another variable to be involved. We do know that, firstly, the most effective clinical treatment for PMDD currently is the use of selective serotonin reuptake inhibitors (SSRIs), and that, secondly, estradiol interacts with the serotonergic system. This is why we hypothesize that women suffering from PMDD have a more pronounced sensitivity of the serotonergic system to physiological fluctuations in estradiol. Through the use of functional and structural magnetic resonance imaging (MRI) and PET, we tested if estradiol fluctuations are associated with depressed mood in women suffering from PMDD, and whether this relationship is moderated by changes in serotonin transporter binding. This study provides the first quantitative neurochemical dataset in vivo to identify a mechanistic biomarker for a newly recognized psychiatric disorder. ${ }^{28}$

As not all women will experience depressive symptoms associated with these endogenous sex hormone fluctuations, it is also critical to build highly characterized, well-controlled models in healthy controls to better understand how subtle fluctuations in endogenous hormones may already influence brain microstructure and structural connectivity. In an exemplary

25 American Psychiatric Association, 2013.

26 Frokjaer/Pinborg/Holst/Overgaard/Henningsson/Heede/Larsen/Jensen/Agn/Nielsen, Role of Serotonin Transporter Changes in Depressive Responses to Sex-Steroid Hormone Manipulation: A Positron Emission Tomography Study, in: Biological psychiatry 2015, 534-43.

27 Bäckström/Andreen/Birzniece/Björn/Johansson/Nordenstam-Haghjo/Nyberg/SundströmPoromaa/Wahlström/Wang, The Role of Hormones and Hormonal Treatments in Premenstrual Syndrome, in: CNS drugs 2003, 325-42.

28 Association, 2013; Reed/First/Kogan/Hyman/Gureje/Gaebel/Maj/Stein/Maercker/Tyrer, 2019. 
study, Barth and colleagues ${ }^{29}$ measured gray matter density changes and white matter microstructure in a single woman across the entire menstrual cycle (30 time-points). The authors found that estradiol levels correlated with both measures in the hippocampus on a day-by-day timescale, suggesting a remarkable degree of hippocampal plasticity in response to sex hormone fluctuations. As this study demonstrated the feasibility and importance of a longitudinal MRI study design to test joint dynamics of the menstrual cycle and the brain, we have now extended this study through the use of ultra-high field 7-Tesla MRI to assess subtle changes in hippocampal subfield volume, white matter microstructure, resting state activity, and endogenous sex hormone fluctuations in a larger sample of women at six critical time-points across the menstrual cycle.

In addition to studying specific hormonal transition states in pathology and in health, another broader approach is to assess sex differences in risk trajectories for neurodegenerative and neuropsychiatric risk across the lifespan. In this third line of work, Zsido and colleagues ${ }^{30}$ investigated associations between structural patterns of brain aging, visceral fat as a metabolic risk factor for structural brain atrophy, and estradiol levels in 473 women and 501 men (19-79 years old). The study found that visceral fat was associated with compromised brain network structure and worse cognitive performance in both men and women, but that estradiol may protect the brain against these structural patterns of atrophy in women only. The authors also observed that women appeared to accumulate the risk factor of visceral fat at the fastest rate during midlife-a time window when estradiol levels rapidly fluctuate and decrease in women during perimenopause. As visceral fat increases and estradiol decreases during the midlife transition, perimenopause may represent a window of increased risk for cognitive health and thus a critical time for possible prevention of late-life cognitive decline. To further explore this sex-specific finding, the authors conducted a follow-up analysis in the midlife female cohort and found that, although the groups did not differ by age or visceral fat levels, women with lower estradiol levels displayed less healthy patterns of brain structure and weaker memory performance. A complementary study ${ }^{31}$ reported an interaction between unfavorable metabolic states, sex hormones, and menopause status, indicating that elevated testosterone levels and

29 Barth/Steele/Mueller/Rekkas/Arélin/Pampel/Burmann/Kratzsch/Villringer/Sacher, 2016.

30 Zsido/Heinrich/Slavich/Beyer/Masouleb/Kratzsch/Raschpichler/Mueller/Scharrer/ Löfler, 2019.

31 Stanikova/Zsido/Luck/Pabst/Enzenbach/Bae/Thiery/Ceglarek/Engel/Wirkner, 2019.

https://doi.org/10.5771/9783748924869-27, am 26.04.2023, 17:02:00 
changes in body weight have differential effects on depression susceptibility depending on whether a woman is pre-menopause or post-menopause. These findings have important clinical implications for developing sex-specific strategies to promote a healthy cognitive and brain aging trajectory.

A central aim of the EGG lab is not only to understand individual depression risk but also to inform and improve relevant clinical strategies. A current project includes a longitudinal model of how selective serotonin reuptake inhibitors (SSRIs), the first-line treatment for the majority of patients with depression and anxiety, influences brain activity and neurochemistry. Over half of major depressive disorder patients have inadequate responses to initial antidepressant therapy, ${ }^{32}$ leading to weeks of trial and error in an attempt to find the right medication. Given that women are twice as likely to suffer from depression as men are, ${ }^{33}$ as well as the highly variable response rates and reported sex differences in response to SSRIs, ${ }^{34}$ there is a critical need to understand the electrophysiological mechanisms underlying SSRI action in women and identify a neurophysiological biomarker to predict individual responsivity to SSRI intake. We administered a commonly-prescribed, fast-acting, clinically-relevant dose of $20 \mathrm{mg}$ escitalopram for one week to assess how escitalopram affects brain function, cortical excitatory/inhibitory balance, and neurochemical shifts in the healthy brain using multimodal techniques: functional and structural MRI, electroencephalography (EEG), and MR spectroscopy, respectively. Given the continuously rising number of prescribed antidepressants ${ }^{35}$ and the increased risk of depression in women worldwide, establishing this model in healthy women provides a well-timed preclinical framework for future translational research in clinical populations and a crucial next step towards informing pharmacological treatment strategies at an individual level.

Depression is a multifaceted disease. Sex hormones are not the sole contributing factor but rather part of a complex and dynamic interplay of neurotransmitters, metabolic risk factors, hormones, psychosocial stress, and possibly an inflammatory response. ${ }^{36}$ Additionally, while the studies mentioned thus far discussed fluctuations in endogenous hormones, it is also known that emotion and mood are influenced by exogenous manipulations

32 Bschor/Kern/Henssler/Baethge, 2016.

33 Kessler/Berglund/Demler/Jin/Koretz/Merikangas/Rush/Walters/Wang, 2003.

34 Gaynes/Warden/Trivedi/Wisniewski/Fava/Rush, 2009; LeGates/Kvarta/Thompson, 2019; Bschor/Kern/Henssler/Baethge, 2016.

35 Iacobucci, 2019.

36 Slavich/Sacher, 2019. 
of sex hormones, such as the use of oral contraceptives. ${ }^{37}$ The essential message is that, when studying risk trajectories for neurodegenerative and neuropsychiatric disorders, particularly those with prominent sex differences in risk rates, it is important to investigate sex differences and the role of sex hormonal environment. This can be through analyzing sex as a primary variable in a half-male half-female population, or in a single-sex study explicitly designed to further delineate a previously observed sex-specific effect or sex-specific experience (e.g., PMDD, pregnancy, oral contraceptive use, menopause).

Recognition of the importance of sex and gender is contributing to a paradigm shift in the neurosciences. The National Institute of Health $(\mathrm{NIH})$ released their first Research Project Grant on sex and gender in November 2019 and leading scientists are defining how female brain health warrants its own scientific discipline. ${ }^{38}$ Major scientific journals such as The Lancet, Nature and Science are increasing discussions surrounding the consequences of missing female representation in scientific research, creating more inclusive editorial policies and expectations for study designs, and dedicating entire issues and projects to sex- and gender-specific advances in scientific discovery. ${ }^{39}$ Finally, research on academic inclusion and success is emerging to address gender imbalance in who gets funded, invited as peer reviewers, and cited in neuroscience research. ${ }^{40}$ In conclusion, robust evidence for sex differences in the neurobiology of the brain exists. ${ }^{41}$ The topic is sensitive and controversially discussed because such findings are often misused to perpetuate gender stereotypes and sexist beliefs. Gender equality and lifting barriers for women in our society are ethical imperatives, and not causally linked to the presence or absence of biological sex differences. Attempts to root gender equality in biology are therefore misguided. Not actively pursuing this line of research will hinder scientific discovery and can be detrimental to the quality of healthcare for women. We encourage the investigation of sex and gender as variables of interest and the use of this knowledge as an opportunity to increase gender equality in the basic sciences and in clinical application.

37 Lewis/Kimmig/Zsido/Jank/Derntl/Sacher, 2019.

38 Galea, 2019.

39 Shansky, 2019; Mogil, 2020; Editorial in Nature, 2020; Editorial in The Lancet, 2020; Takehiro/Iwasaki, 2021.

40 National Institutes of Health, 2020; Dworkin/Linn/Teich/Zurn/Shinohara/Bassett, 2020; Chawla, 2018.

41 Liu/Seidlitz/Blumenthal/Clasen/Raznahan, 2020. 


\section{REFERENCES}

American Psychiatric Association. (2013). Diagnostic and Statistical Manual of Mental Disorders (DSM-5®): American Psychiatric Pub.

Bäckström, Torbjörn, Lotta Andreen, Vita Birzniece, Inger Björn, Inga-Maj Johansson, Maud Nordenstam-Haghjo, Sigrid Nyberg, Inger Sundström-Poromaa, Göran Wahlström, and Mingde Wang. (2003). The role of hormones and hormonal treatments in premenstrual syndrome. CNS Drugs, 17(5), 325-342.

Barth, Claudia, Christopher J Steele, Karsten Mueller, Vivien P Rekkas, Katrin Arélin, Andre Pampel, Inga Burmann, Jürgen Kratzsch, Arno Villringer, and Julia Sacher. (2016). In-vivo dynamics of the human hippocampus across the menstrual cycle. Scientifc Reports, 6, 32833.

Beery, Annaliese K, and Irving Zucker. (2011). Sex bias in neuroscience and biomedical research. Neuroscience \& Biobehavioral Reviews, 35(3), 565-572.

Bromet, Evelyn, Laura Helena Andrade, Irving Hwang, Nancy A Sampson, Jordi Alonso, Giovanni De Girolamo, Ron De Graaf, Koen Demyttenaere, Chiyi Hu, and Noboru Iwata. (2011). Cross-national epidemiology of DSM-IV major depressive episode. BMC Medicine, 9(1), 90.

Bschor, Tom, Hannah Kern, Jonathan Henssler, and Christopher Baethge. (2016). Switching the antidepressant after nonresponse in adults with major depression: a systematic literature search and meta-analysis. The Journal of Clinical Psychiatry, $79(1)$.

Carter, Christine L, Eileen M Resnick, Monica Mallampalli, and Anna Kalbarczyk. (2012). Sex and gender differences in Alzheimer's disease: recommendations for future research. Journal of Women's Health, 21(10), 1018-1023.

Chakravarty, Dimple, Sujit S Nair, Nada Hammouda, Parita Ratnani, Yasmine Gharib, Vinayak Wagaskar, Nihal Mohamed, Dara Lundon, Zachary Dovey, and Natasha Kyprianou. (2020). Sex differences in SARS-CoV-2 infection rates and the potential link to prostate cancer. Communications Biology, 3(1), 1-12.

Chawla. Nature 561, 295-296 (2018) doi: https://doi.org/10.1038/d41586-018-066786.

Chen, Nanshan, Min Zhou, Xuan Dong, Jieming Qu, Fengyun Gong, Yang Han, Yang Qiu, Jingli Wang, Ying Liu, and Yuan Wei. (2020). Epidemiological and clinical characteristics of 99 cases of 2019 novel coronavirus pneumonia in Wuhan, China: a descriptive study. The Lancet, 395(10223), 507-513.

Cirillo, Davide, Silvina Catuara-Solarz, Czuee Morey, Emre Guney, Laia Subirats, Simona Mellino, Annalisa Gigante, Alfonso Valencia, María José Rementeria, and Antonella Santuccione Chadha. (2020). Sex and gender differences and biases in artificial intelligence for biomedicine and healthcare. NPJ Digital Medicine, 3(1), 1-11.

Deecher, Darlene, Terrance H Andree, Diane Sloan, and Lee E Schechter. (2008). From menarche to menopause: exploring the underlying biology of depression in women experiencing hormonal changes. Psychoneuroendocrinology, 33(1), 317. 
D'Ignazio, Catherine, Lauren F. Klein. (2020). “Data Feminism”, MIT Press.

Dworkin, J.D., Linn, K.A., Teich, E.G. et al. The extent and drivers of gender imbalance in neuroscience reference lists. Nat Neurosci 23, 918-926 (2020). https:// doi.org/10.1038/s41593-020-0658-y.

Editorial in Nature. (2020). https://doi.org/10.1038/d41586-020-03459-y.

Editorial in The Lancet. (2020). https:/www.thelancet.com/journals/lancet/article/ PIIS0140-6736(19)33170-8/fulltext.

Freeman, Ellen W, Mary D Sammel, David W Boorman, and Rongmei Zhang. (2014). Longitudinal pattern of depressive symptoms around natural menopause. JAMA Psychiatry, 71(1), 36-43.

Frokjaer, Vibe Gedsoe, Anja Pinborg, Klaus Kähler Holst, Agnete Overgaard, Susanne Henningsson, Maria Heede, Elisabeth Clare Larsen, Peter Steen Jensen, Mikael Agn, and Anna Pors Nielsen. (2015). Role of serotonin transporter changes in depressive responses to sex-steroid hormone manipulation: a positron emission tomography study. Biological Psychiatry, 78(8), 534-543.

Galea, Liisa A. (2019, July 31). I Should Be Allowed to Leave Men Out of My Studies. Retrieved December 2, 2020, from https://www.vice.com/en_ca/article/ j5wy7x/i-should-be-allowed-to-leave-men-out-of-my-studies.

Galea, Liisa AM, Elena Choleris, Arianne YK Albert, Margaret M McCarthy, and Farida Sohrabji. (2020). The promises and pitfalls of sex difference research. Frontiers in Neuroendocrinology, 56, 100817.

Gaynes, Bradley N, Diane Warden, Madhukar H Trivedi, Stephen R Wisniewski, Maurizio Fava, and A John Rush. (2009). What did STAR* D teach us? Results from a large-scale, practical, clinical trial for patients with depression. Psychiatric Services, 60(11), 1439-1445.

Global Health 5050. (2020). COVID-19 sex-disaggregated data tracker: Sex, gender and COVID-19. Retrieved December 2, 2020, from https://globalhealth5050.org/ covid19/sex-disaggregated-data-tracker.

Guan, Wei-jie, Zheng-yi Ni, Yu Hu, Wen-hua Liang, Chun-quan Ou, Jian-xing He, Lei Liu, Hong Shan, Chun-liang Lei, and David SC Hui. (2020). Clinical characteristics of coronavirus disease 2019 in China. New England Journal of Medicine, 382(18), 1708-1720.

Iacobucci, Gareth. (2019). NHS prescribed record number of antidepressants last year. Bmj, 364, 11508.

Kessler, Ronald C, Patricia Berglund, Olga Demler, Robert Jin, Doreen Koretz, Kathleen R Merikangas, A John Rush, Ellen E Walters, and Philip S Wang. (2003). The epidemiology of major depressive disorder: results from the National Comorbidity Survey Replication (NCS-R). Jama, 289(23), 3095-3105.

Larrazabal, Agostina J, Nicolás Nieto, Victoria Peterson, Diego H Milone, and Enzo Ferrante. (2020). Gender imbalance in medical imaging datasets produces biased classifiers for computer-aided diagnosis. Proceedings of the National Academy of Sciences, 117(23), 12592-12594.

LeGates, Tara A, Mark D Kvarta, and Scott M Thompson. (2019). Sex differences in antidepressant efficacy. Neuropsychopharmacology, 44(1), 140-154. 
Lewis, Carolin A, Ann-Christin S Kimmig, Rachel G Zsido, Alexander Jank, Birgit Derntl, and Julia Sacher. (2019). Effects of hormonal contraceptives on mood: A focus on emotion recognition and reactivity, reward processing, and stress response. Current Psychiatry Reports, 21(115).

Light, Karen Patricia, Anthony Timothy Lovell, Hisham Butt, Nicholas John Fauvel, and Anita Holdcroft. (2006). Adverse effects of neuromuscular blocking agents based on yellow card reporting in the UK: are there differences between males and females? Pharmacoepidemiology and Drug Safety, 15(3), 151-160.

Liu, Siyuan, Jakob Seidlitz, Jonathan D. Blumenthal, Liv S. Clasen, and Armin Raznahan. (2020). Integrative structural, functional, and transcriptomic analyses of sex-biased brain organization in humans. Proceedings of the National Academy of Sciences, 201919091. doi:10.1073/pnas.1919091117

Mersha, Tesfaye B, Lisa J Martin, Jocelyn M Biagini Myers, Melinda Butsch Kovacic, Hua He, Mark Lindsey, Umasundari Sivaprasad, Weiguo Chen, and Gurjit K Khurana Hershey. (2015). Genomic architecture of asthma differs by sex. Genomics, 106(1), 15-22.

Mogil, Jeffrey S. (2020). Qualitative sex differences in pain processing: emerging evidence of a biased literature. Nature Reviews Neuroscience, 1-13.

Multiple Risk Factor Intervention Trial Research Group. (1982). Multiple Risk Factor Intervention Trial: risk factor changes and mortality results. Jama, 248, 14651477.

National Institutes of Health. (2020). NIH Data Book: Data by Gender. https://repo rt.nih.gov/nihdatabook/category/16.

Nott, PN, M Franklin, C Armitage, and MG Gelder. (1976). Hormonal changes and mood in the puerperium. The British Journal of Psychiatry, 128(4), 379-383.

O'hara, Michael W, and Annette M Swain. (1996). Rates and risk of postpartum depression-a meta-analysis. International Review of Psychiatry, 8(1), 37-54.

O'Hara, Michael W, Janet A Schlechte, David A Lewis, and Ellen J Wright. (1991). Prospective study of postpartum blues: biologic and psychosocial factors. Archives of general psychiatry, 48(9), 801-806.

Park, Matthew D. (2020). Sex differences in immune responses in COVID-19. Nature Reviews Immunology, 20(461).

Perez, Caroline Criado. (2019). Invisible Women: Exposing Data Bias in a World Designed for Men. Random House.

Pirastu, Nicola, Mattia Cordioli, Priyanka Nandakumar, Gianmarco Mignogna, Abdel Abdellaoui, Ben Hollis, Masahiro Kanai, Veera Manikandan Rajagopal, Pietro Della Briotta Parolo, and Nikolas Baya. (2020). Genetic analyses identify widespread sex-differential participation bias. bioRxiv. doi: https:/doi.org/ 10.1101/2020.03.22.001453

Reed, Geoffrey M, Michael B First, Cary S Kogan, Steven E Hyman, Oye Gureje, Wolfgang Gaebel, Mario Maj, Dan J Stein, Andreas Maercker, and Peter Tyrer. (2019). Innovations and changes in the ICD-11 classification of mental, behavioural and neurodevelopmental disorders. World Psychiatry, 18(1), 3-19. 
Ruitenberg, Annemieke, Alewijn Ott, John C van Swieten, Albert Hofman, and Monique MB Breteler. (2001). Incidence of dementia: does gender make a difference? Neurobiology of aging, 22(4), 575-580.

Sacher, Julia, Alan A Wilson, Sylvain Houle, Pablo Rusjan, Sabrina Hassan, Peter M Bloomfield, Donna E Stewart, and Jeffrey H Meyer. (2010). Elevated brain monoamine oxidase A binding in the early postpartum period. Archives of general psychiatry, 67(5), 468-474.

Sacher, Julia, P Vivien Rekkas, Alan A Wilson, Sylvain Houle, Leslie Romano, Jinous Hamidi, Pablo Rusjan, Ian Fan, Donna E Stewart, and Jeffrey H Meyer. (2015). Relationship of monoamine oxidase-A distribution volume to postpartum depression and postpartum crying. Neuropsychopharmacology, 40(2), 429.

Shansky, Rebecca M. (2019). Are hormones a "female problem" for animal research? Science, 364(6443), 825-826.

Sharma, Garima, Annabelle Santos Volgman, and Erin D Michos. (2020). Sex Differences in Mortality from COVID-19 Pandemic: Are Men Vulnerable and Women Protected? JACC: Case Reports.

Shock, Nathan W. (1984). Normal Human Aging: The Baltimore Longitudinal Study of Aging. US Government Printing Office.

Simon, Viviana. (2005). Wanted: women in clinical trials. Science, 308(5728), 15171518.

Slavich, George M, and Julia Sacher. (2019). Stress, sex hormones, inflammation, and major depressive disorder: Extending Social Signal Transduction Theory of Depression to account for sex differences in mood disorders. Psychopharmacology, 236, 3063-3079.

Stanikova, Daniela, Rachel G Zsido, Tobias Luck, Alexander Pabst, Cornelia Enzenbach, Yoon Ju Bae, Joachim Thiery, Uta Ceglarek, Christoph Engel, and Kerstin Wirkner. (2019). Testosterone imbalance may link depression and increased body weight in premenopausal women. Translational psychiatry, 9(1), 160.

Steering Committee of the Physicians' Health Study Research Group*. (1989). Final report on the aspirin component of the ongoing Physicians' Health Study. New England Journal of Medicine, 321(3), 129-135.

Sugimoto, Cassidy R, Yong-Yeol Ahn, Elise Smith, Benoit Macaluso, and Vincent Larivière. (2019). Factors affecting sex-related reporting in medical research: a cross-disciplinary bibliometric analysis. The Lancet, 393(10171), 550-559.

Takehiro/Iwasaki, 2021, https://doi.org/10.1126/science.abe7199, "Sex differences in immune responses", published in Science.

Tharpe, Nell. (2011). Adverse drug reactions in women's health care. Journal of Midwifery \& Women's Health, 56(3), 205-213.

Wenham, Clare, Julia Smith, and Rosemary Morgan. (2020). COVID-19: the gendered impacts of the outbreak. The Lancet, 395(10227), 846-848.

Will, Tyler R, Stephanie B Proaño, Anly M Thomas, Lindsey M Kunz, Kelly C Thompson, Laura A Ginnari, Clay H Jones, Sarah-Catherine Lucas, Elizabeth M Reavis, and David M Dorris. (2017). Problems and progress regarding sex bias and omission in neuroscience research. eneuro, 4(6). 
Zsido, Rachel G, Arno Villringer, and Julia Sacher. (2017). Using positron emission tomography to investigate hormone-mediated neurochemical changes across the female lifespan: implications for depression. International Review of Psychiatry, 29(6), 580-596.

Zsido, Rachel G, Matthias Heinrich, George M Slavich, Frauke Beyer, Shahrzad Kharabian Masouleh, Juergen Kratzsch, Matthias Raschpichler, Karsten Mueller, Ulrike Scharrer, Markus Löffler, Matthias L Schroeter, Michael Stumvoll, Arno Villringer, A Veronica Witte, and Julia Sacher. (2019). Association of Estradiol and Visceral Fat With Structural Brain Networks and Memory Performance in Adults. JAMA Network Open, 2(6), e196126-e196126.

\section{ABOUT THE AUTHORS}

\section{Rachel Zsido}

Rachel Zsido is a PhD student at the Max Planck Institute for Human Cognitive and Brain Sciences and a founding member of her institute's Diversity Committee. She is enrolled in the Max Planck School of Cognition and the International Max Planck Research School NeuroCom graduate programs. She completed her undergraduate training at Harvard University and Massachusetts General Hospital, where she studied the influence of ovarian hormones on psychological and neural correlates of fear conditioning and extinction in women suffering from anxiety and posttraumatic stress disorder. Her current research focuses on how ovarian hormones and the serotonergic system interact to influence brain microstructure and neurochemistry, and the implications these interactions have on depression susceptibility, resilience, and treatment. Her doctoral work has received prizes from three international conferences, as well as a fellowship from the Joachim Herz Foundation.

\section{Selected Publications:}

Zsido, Rachel G, Matthias Heinrich, George M Slavich, Frauke Beyer, Shahrzad Kharabian Masouleh, Juergen Kratzsch, Matthias Raschpichler, Karsten Mueller, Ulrike Scharrer, Markus Löffler, Matthias L Schroeter, Michael Stumvoll, Arno Villringer, A Veronica Witte, and Julia Sacher. (2019). Association of Estradiol and Visceral Fat With Structural Brain Networks and Memory Performance in Adults. JAMA Network Open, 2(6), e196126. https://doi.org/10.1001/jamanetworkopen.2019.6126 
Stanikova, Daniela, Rachel G Zsido, Tobias Luck, Alexander Pabst, Cornelia Enzenbach, Yoon Ju Bae, Joachim Thiery, Uta Ceglarek, Christoph Engel, and Kerstin Wirkner. (2019). Testosterone imbalance may link depression and increased body weight in premenopausal women. Translational Psychiatry, 9(1), 1-12. https://doi.org/10.1038/s41398-019-0487-5

Zsido, Rachel G, Arno Villringer, and Julia Sacher. (2017). Using positron emission tomography to investigate hormone-mediated neurochemical changes across the female lifespan: implications for depression. International Review of Psychiatry, 29(6), 580-596. https://doi.org/10.1080/09540261.2017.1397607

\section{Julia Sacher}

Dr. Julia Sacher is a psychiatrist and neuroscientist, and leads the Minerva Research Group: Emotion Neuroimaging (EGG) Lab at the Max Planck Institute for Human Cognitive and Brain Sciences in Leipzig, Germany. She obtained her MD and PhD degrees from the Medical University of Vienna in 2004 and 2007, and was a postdoctoral fellow at the Centre of Addiction and Mental Health (CAMH), University of Toronto until 2009. Dr Sacher and her group study how endogenous hormonal changes affect the human brain and behavior and aim to understand the unique vulnerabilities of women to neurodegenerative diseases, such as depression and dementia. Dr Sacher has pioneered a neurobiological model for postpartum blues, her work has been published in top-ranking journals, such as JAMA Psychiatry, Biological Psychiatry, Neuropsychopharmacology and Current Biology and she has won international fellowship grants and awards, such as the Humboldt Fellowship, a CIHR fellowship, the Branco Weiss Society in Science fellowship, the CINP Rafaelsen Award, and two Young NARSAD Investigator Awards (Brain and Behavior Foundation).

\section{Selected Publications:}

Slavich, George M, and Julia Sacher. (2019). Stress, sex hormones, inflammation, and major depressive disorder: Extending Social Signal Transduction Theory of Depression to account for sex differences in mood disorders. Psychopharmacology, 236, 3063-3079. doi: 10.1007/s00213-019-05326-9.

Sacher, Julia, P Vivien Rekkas, Alan A Wilson, Sylvain Houle, Leslie Romano, Jinous Hamidi, Pablo Rusjan, Ian Fan, Donna E Stewart, and Jeffrey H Meyer. (2015). Relationship of monoamine oxidase-A distribution volume to postpartum depression and postpartum crying. Neuropsychopharmacology, 40(2), 429435. https://doi.org/10.1038/npp.2014.190

Schaefer, A., Burmann, I., Regenthal, R., Arélin, K., Barth, C., Pampel, A., ... Sacher, J. (2014). Serotonergic modulation of intrinsic functional connectivity. Current Biology, 24(19), 2314-2318. https://doi.org/10.1016/j.cub.2014.08.024 


\section{ABOUT THE INSTITUTE}

Max Planck Institute for Human Cognitive and Brain Sciences, Emotion \& Neuroimaging Lab

Research at the Max Planck Institute for Human Cognitive and Brain Sciences revolves around human cognitive abilities and cerebral processes, with a focus on the neural basis of brain functions such as language, emotions, human social behavior, music and action. Through the use of multimodal neuroimaging techniques, such as Positron Emission Tomography (PET), Magnetic Resonance Imaging (MRI) and electroencephalography (EEG), the Emotion \& Neuroimaging (EGG) Lab, an independent research group, led by Dr Sacher, investigates how sex hormones affect brain and behavior across the adult lifespan and strives to identify the mechanisms underlying the unique vulnerabilities of women to depression and dementia. 